\title{
Update
}

Ophthalmologica

\section{Choices in Correction of Aphakia during Vitrectomy}

\author{
Andrew Bastawrous Craig Parkes Som Prasad \\ Department of Ophthalmology, Wirral University Hospital, Upton, UK
}

\section{Key Words}

Aphakia · Intraocular lens implant, secondary • Anterior chamber intraocular lens · Posterior chamber intraocular lens · Intraocular lens, sclera-fixated · Capsular support, inadequate

\begin{abstract}
Phacoemulsification with an intraocular lens implant is a safe and effective means of correction of visual loss from cataract. The high frequency of this procedure world wide means inevitable direct surgical complications will be numerous even though infrequent. The approach to correcting aphakia following complicated cataract surgery is dependent on multiple factors including the degree to which the capsular bag is intact and the type of intraocular lens that has been inserted. We discuss several approaches and strategies for managing dropped intraocular lenses, the correction of aphakia and the safety and longterm results of anterior chamber intraocular lens placement.

Copyright $\odot 2011$ S. Karger AG, Basel
\end{abstract}

\section{Introduction}

Cataract extraction and intraocular lens (IOL) implantation are arguably two of the most important advances in modern ophthalmology. The now combined procedure of phacoemulsification and IOL implantation is a safe and effective means of restoring vision loss from cataract. Although capsular rupture and zonular dialysis are infrequent complications of phacoemulsification and IOL implant surgery, the high volume and demand for cataract surgery make these complications numerous enough for ophthalmic surgeons to possess strategies to allow successful management of complicated surgery and restore visual loss secondary to aphakia. Approximately 300,000 people annually have cataract surgery in the UK, with a posterior capsule rupture and/or vitreous loss rate of $1.92 \%$ and simple zonular dialysis rate of $0.46 \%$ [1]; nuclear fragments may be displaced into the vitreous in 2-3 per 1,000 operations [2]. In the British Ophthalmic Surveillance Unit study of 2003-2004, up to three quarters of IOL placed at primary surgery were exchanged or explanted at vitrectomy for removal of nuclear fragments [3].

In recent years surgical techniques have evolved to deal with these situations. This review will focus on current strategies for managing a dropped IOL, correction of aphakia in the absence of adequate capsular support, and the safety and long-term results of anterior chamber IOL (ACIOL).

\section{KARGER \\ Fax +4161306 1234 \\ E-Mail karger@karger.ch}

www.karger.com
(C) 2011 S. Karger AG, Basel

0030-3755/11/2265-0046\$38.00/0

Accessible online at: www.karger.com/oph
Mr. Som Prasad, MS, FRCSEd, FRCOphth, FACS

Department of Ophthalmology

Arrowe Park Hospital, Arrowe Park Road

Upton CH49 5PE (UK)

E-Mailsprasad@rcsed.ac.uk 
Fig. 1. Decision tree for management of subluxated or dislocated IOL. CCC $=$ Curvilinear capsulorhexis.

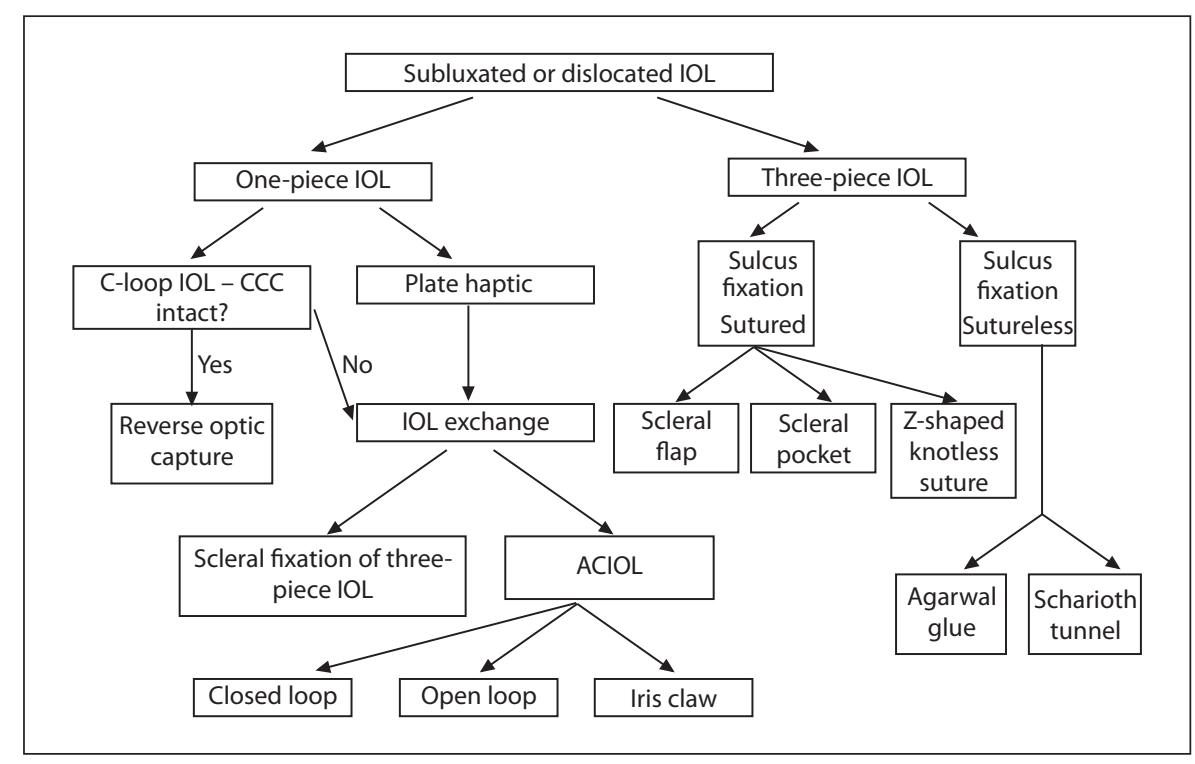

\section{Dropped IOL}

An IOL can drop into the vitreous cavity due to trauma, zonular dehiscence or posterior capsular rupture. These situations can arise when an IOL is placed without secure support, but also as a long-term complication due to progressive zonular loss following the initial insult. Removal requires a complete pars plana vitrectomy; care must be taken to remove as much of the vitreous as possible, first concentrating on removing all peripheral vitreous, and only then is any vitreous entangled with the IOL dealt with, before attempts to move the IOL are made. Most of these eyes will have a posterior vitreous detachment already, but this should be confirmed. Perfluorocarbon liquid can be used to cover the macula and shield it from trauma from a part of the IOL whilst it is being manoeuvred. A full assessment of the capsule can then be made as this will guide the surgeon's choice of lens placement and method of fixation, and this is later discussed in detail. Using end-gripping forceps, the IOL is lifted into the midvitreous cavity, and if the IOL is still inside the capsular bag complex, this may need to be cleaned off the lens with the vitrectomy cutter. If the lens is in good condition and is of a type suitable for ciliary sulcus placement, then this can be fixed in place using one of the methods described below. If the lens needs replacing, then it is passed into the anterior chamber, temporarily placed in front of the iris. A self-sealing corneal incision is then fashioned and the IOL cut in half and removed.
For rigid lenses a scleral tunnel offers a self-sealing incision, allowing surgery to proceed in a 'closed-chamber' system. Particular attention needs to be given to the IOL design chosen either if an ACIOL is being considered or sulcus placement is planned. Chang et al. [4] reported the complications associated with sulcus placement of singlepiece acrylic lenses. They attribute the thicker haptic size, squared optic edges and hydrophobic 'tacky' acrylic surface to increased risk of iris chafing causing pigment dispersion, uveitis and secondary glaucoma. The haptics are planar rather than angulated, and often the overall haptic dimension is smaller than ideal in a situation where perfect centration may not always be possible in the long term. We advocate IOL exchange if the dislocated lens is a single-piece acrylic lens. Finally after the lens has been fixated, it is necessary to ensure complete removal of the perfluorocarbon liquid (if used) and careful inspection of the peripheral retina for potential iatrogenic retinal tears. It is particularly important to inspect the vitreous base as tiny retinal breaks close to the ora serrata are easy to miss but can cause subsequent retinal detachments.

\section{IOL Fixation Strategies}

We now discuss the various methods of IOL fixation both with and without capsular support, depending on the type of IOL already in situ and if it is appropriate to use the same IOL (fig. 1). 


\section{C-Loop IOL with Intact Anterior Curvilinear Capsulorhexis}

An anterior curvilinear capsulorhexis (CCC) provides a tear-resistant opening that allows phacoemulsification of the cataract and secure IOL implantation in the capsular bag [5]. When surgery is complicated by a large posterior capsular tear and resultant inadequate capsular support for in-the-bag IOL implantation, but an intact anterior CCC remains, it is possible to place a sulcus IOL with optic capture. Advantages to this technique are the prevention of uveal tissue chaffing and secure IOL placement. The CCC must be intact and smaller than the optic diameter of the IOL. In case future pars plana vitrectomy is required for any reason, these IOL do not destabilise in spite of deep scleral indentation intraoperatively; presumably they remain stable in the face of other insults as well. Once adequate anterior vitrectomy has been performed with care to avoid extension of the capsular tear, the IOL can be placed into the ciliary sulcus. The optic is placed through the CCC with gentle pressure, one side at a time. The haptics remain within the sulcus, with the optic captured posteriorly through the CCC into the capsular bag. Other variants of the lens optic capture technique have been described [6], but the one described above remains the most useful, which can often be deployed to good effect.

\section{Three-Piece IOL}

In the scenario where a three-piece IOL is found to have decentred but is still within the bag due to zonular dialysis, it is possible to secure one of the haptics to the sclera to centre the optic. If the eye is aphakic and a posterior chamber IOL (PCIOL) is to be inserted without adequate capsular support, then both haptics are required to be secured to the sclera. We prefer a PCIOL in most circumstances because there are multiple advantages to placing the IOL in the posterior chamber: being at or close to the eye's nodal point has optical benefits, and its safe distance from the trabecular meshwork and corneal endothelium possibly reduces the risk of glaucoma, uveitis and corneal decompensation and, if well secured, iris chaffing. A PCIOL may be secured in the ciliary sulcus by various surgical techniques which we discuss below.

\section{Ciliary Sulcus, Suture-Fixated IOL}

There are multiple techniques for achieving a secure transscleral fixated PCIOL with variations in suture material, method of securing of the haptics, needle passing (ab externo or ab interno) and knot burial. If secondary IOL implantation is required, then there is also a multitude of IOLs on the market specifically designed for sutured fixation. You can choose between foldable and nonfoldable ones, and two, three or four holes or eyelets for suture fixation.

With all methods we recommend starting by doing a triamcinolone-assisted anterior vitrectomy, which can be performed either via a pars plana approach or via a corneoscleral wound, depending on surgeon preference or experience. The advantage of the pars plana vitrectomy approach is its ability to maintain good ocular pressure whilst passing the scleral suture, thereby increasing the chance of good suture placement within the ciliary sulcus [4]. The same accuracy can be obtained by closing the corneoscleral wound following anterior vitrectomy or using an anterior chamber maintainer.

Increased suture fixation points allow for better IOL stability; however, this must be weighed against a potential risk of bleeding, inflammation and suture erosion. We therefore recommend making a thorough assessment of the capsular support as approximately 3 clock hours can provide sufficient support for one haptic. We have chosen to describe three specific suturing methods in detail, but alternative methods have been described.

\section{Scleral Flap}

One of the main considerations using suture-fixated lenses is the risk of endophthalmitis and suture erosion. In an attempt to minimise this risk, partial-thickness triangular scleral flaps can be made at 4 and 10 o'clock to avoid the long posterior ciliary arteries associated with the 3-and 9-o'clock positions, decreasing the risk of haemorrhage. An anterior local conjunctival peritomy is necessary, then fashioning two $3 \times 2 \mathrm{~mm}$ partial-thickness scleral flaps $180^{\circ}$ apart. A straight needle attached to a 9-0 polypropylene suture is then passed ab externo 1.5 $\mathrm{mm}$ posterior to the surgical limbus, parallel to the iris. On the opposite side, a 28 -gauge hollow needle is passed ab externo $1.5 \mathrm{~mm}$ from the limbus, and the suture needle is retrieved through it. A corneal wound is made if not already existing, its size depending on the whether the chosen lens is designed to be folded or not. A loop of suture is retrieved through the corneal wound, cut and attached to the lens haptics, and the lens is inserted. A second 9-0 polypropylene suture is used to take a secure bite just anterior to the existing suture. This is then tied off to both itself and the lens-holding suture. This procedure is repeated at the other scleral bed and then the flaps and conjunctiva are closed. 
Fig. 2. Suture fixation in a scleral pocket. a A peripheral corneal incision like a limbal relaxing incision (LRI) is made (not shown), and then a blade is used to dissect a pocket partial thickness through the sclera. b A needle is passed under the iris and out through the sclera and conjunctiva. A second needle is entered through a similar pass but slightly separated. c The 2 suture arms are external to the conjunctiva. The needles are cut and the suture arms pulled out through the corneal incision (the one resembling an LRI). d As the knot is tied, it buries automatically into the scleral pocket, leaving a minimally disturbed ocular surface.
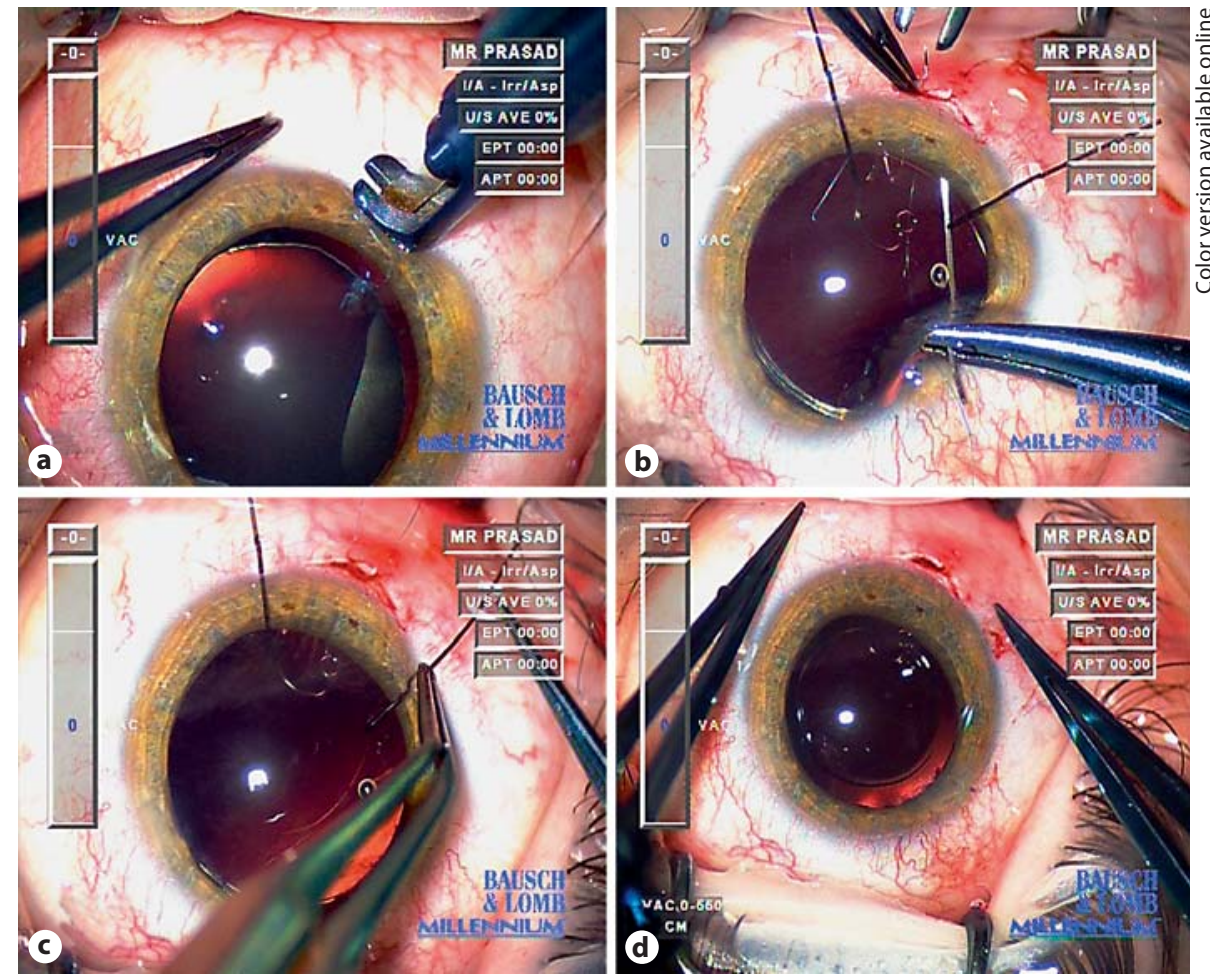

\section{Scleral Pocket}

The scleral pocket is a newer technique described by Hoffman et al. [7] in 2006, allowing for suture burial within the sclera without the need for conjunctival peritomy or preparation of a scleral flap. Two 30-degree peripheral clear corneal incisions are made $180^{\circ}$ apart to a depth of between 300 and $400 \mu \mathrm{m}$ (much like limbal relaxing incisions). Two scleral pockets are dissected posteriorly, roughly $3.0 \mathrm{~mm}$ from the initial incisions. Two 1.0$\mathrm{mm}$ paracenteses are created from the corneal incision into the anterior chamber. The paracentesis can also be used to place a single iris hook exposing the haptics and capsular bag of an unstable or decentred IOL. A 27-gauge hollow needle is then passed $1 \mathrm{~mm}$ posterior to the surgical limbus, through the full thickness of the conjunctiva and the sclera, passing through the dissected pocket and into the ciliary sulcus and then anterior to the haptic/capsular bag complex. A straight needle attached to a 10-0 or 9-0 double-armed polypropylene suture is passed through the corneal paracentesis on the opposite side, docked with the hollow needle and withdrawn through the sclera and conjunctiva. The procedure is repeated, with the second entry site placed 1-2 mm adjacent to the first, $1 \mathrm{~mm}$ from the surgical limbus. This hollow needle is passed posterior to the haptic before angling anteriorly towards the opposite corneal paracentesis, passing through both the posterior and anterior capsular bag before docking with the needle. Alternatively a needle can be passed behind the iris in front of the haptic, and then the other arm behind the haptic (fig. 2).

Once both ends of the suture are through the conjunctiva, the needles can be cut off and the suture ends retrieved from the scleral pocket with a Sinskey hook, or appropriate forceps, one after the other whilst securing the other end with a forceps to prevent pulling the suture through. The suture can now be tied and the knot will be buried in the pocket with no need for pocket closure.

\section{Z-Shaped Knotless Suture}

Szurman et al. [8] described a knotless method of IOL fixation in 2010. This employs the friction of the suture passing through the partial thickness of the sclera. Dr. Szurman has calculated by studies on porcine eyes that 5 passes of the suture through the sclera in a zigzag pattern produce more frictional force than the tensile strength of the 10-0 suture, thereby negating the need for a knot for fixation. This eliminates the risk of knot erosion and reduces the spectre of subsequent endophthalmitis. Perform conjunctival peritomies $180^{\circ}$ apart. A double-ended 10-0 polypropylene suture is passed through the sclera 
$1.3 \mathrm{~mm}$ from the surgical limbus; the authors report that this technique is compatible with any suture method as long as you are left with the suture with the needle still attached. You then make 5 passes of the suture intrasclerally with 5 'indentations' or corners to the zigzag pattern. The suture can then simply be cut and the conjunctival peritomy closed. The advantage of this method is that a single full-thickness pass of the sclera can be incorporated on each side, reducing the bleeding risk, and there is no risk of suture erosion. The method is also relatively quick to perform, taking a little over $1 \mathrm{~min}$ to complete, and the conjunctival peritomy need only be small.

\section{Ciliary Sulcus, Sutureless Fixation of the IOL}

In an attempt to further reduce the potential risks of sulcus-fixated lenses, some authors have identified the sutures as the cause of many of the postoperative complications; these range from persistent uveitis due to iris contact over knot erosions to suture failure. Therefore some surgeons have developed techniques for removing the need for sutures completely, and two of these methods are discussed below.

Scharioth's Sutureless Intrascleral PCIOL Fixation

This technique described by Scharioth et al. [9] is a sutureless method of sulcus fixation of a PCIOL using permanent incarceration of the haptics in a scleral tunnel parallel to the limbus. This technique can be used for sulcus fixation of a secondary PCIOL, but can be used with modification for fixation of a subluxated IOL-capsular bag complex, or for any IOL or intraocular device requiring transscleral fixation. The technique usually involves a pars plana vitrectomy to address pathology resulting from complications of cataract surgery such as dropped lens fragments or indeed IOL, but in other cases posterior vitrectomy is not required. Create a corneal incision suitable for insertion of a three-piece IOL; ideally injector-assisted but foldable three-piece IOL can be used, provided sufficient control of the trailing haptic is maintained. Two 24-gauge straight ab externo sclerotomies are formed using a 24-gauge cannula $1.5-2.0 \mathrm{~mm}$ from the limbus, $180^{\circ}$ from one another. Control the position of the ciliary sulcus sclerotomy; using an indirect viewing system with deep scleral indentation is desirable. Using the 24-gauge cannula, or a 23-gauge MVR blade, prepare two scleral tunnels $180^{\circ}$ from each other. The tunnel is prepared by inserting the cannula parallel to the limbus at $50 \%$ scleral thickness, starting at the sclerotomy insertion point and ending $2.0-3.0 \mathrm{~mm}$ away with externalisation of the cannula. A three-piece IOL is chosen for de- sired postoperative refraction and a haptic-to-haptic diameter equal to the diameter of the ciliary sulcus. Insert the IOL into the eye, ensuring the trailing haptic is within and protruding out of the corneal incision to protect the IOL from dropping into the posterior chamber. Using an end-gripping 25-gauge pair of forceps, pull the leading haptic through the sclerotomy. Then insert the end-gripping forceps into the prepared scleral tunnel from the distal end. Grasp the tip of the leading haptic and pull it into the tunnel. The same procedure is repeated with the trailing haptic. Position the trailing haptic into the eye using suitable forceps. Grasp the tip of the trailing haptic with the 25-gauge forceps through the sclerotomy and position it in the limbus-parallel tunnel. Ensure the optic is centred and the haptics are buried within the tunnels. Close the conjunctiva (fig. 3).

The intermediate follow-up results for this technique have been promising [10]. Sixty-three consecutive patients in 4 institutes performed by 4 surgeons with a median follow-up of 7 months (range: 1-22 months), with 61 of 63 IOL stable and centred ( 2 of 63 decentred). There were no cases of recurrent dislocation, endophthalmitis, retinal detachment or glaucoma.

\section{Agarwal's Fibrin Glue-Assisted Sutureless PCIOL} Implantation

Fibrin glue has been in use to repair cardiac tissue [11] and a sealant in a variety of tissues. This technique describes a sutureless method for PCIOL implantation, utilising scleral flaps associated with tunnels aided by fibrin glue.

Fixate the superior rectus. Perform a conjunctival peritomy and cautery sufficient to create a $2.5 \times 3.0 \mathrm{~mm}$ scleral flap $1.5 \mathrm{~mm}$ from the limbus. The site of these should be chosen to match IOL haptic positioning $180^{\circ}$ to one another. Perform either a vitrectomy via the pars plana or an anterior vitrectomy using an anterior chamber maintainer. If using an anterior chamber maintainer, ensure the entry site does not interfere with scleral pouch positioning. Under the scleral flaps, make two straight sclerotomies using a 22-gauge needle, $1.5 \mathrm{~mm}$ from the limbus. Create a scleral tunnel incision in preparation for IOL introduction. Insert a pair of 25-gauge end-gripping forceps through the inferior sclerotomy and hold it in place to receive the IOL leading haptic as it is introduced through the scleral tunnel. Grasp the tip of the leading haptic and pull it through the inferior sclerotomy, allowing it to follow the curve of the haptic and externalise under the inferior scleral flap. Externalise the trailing haptic through the opposite sclerotomy under the scleral flap. 
Fig. 3. The Scharioth technique. a Subluxated IOL following complicated cataract surgery. b The haptic is grasped by a forceps in the anterior chamber and passed onto a 25-gauge forceps inserted through a sclerostomy $1.5 \mathrm{~mm}$ behind the limbus. The same procedure is repeated with the other haptic at $180^{\circ}$ opposite. c The IOL is centred, with haptics externalised. d A scleral tunnel is created next to the externalised haptic, into which the haptic is buried, and the same procedure is repeated with the other haptic.

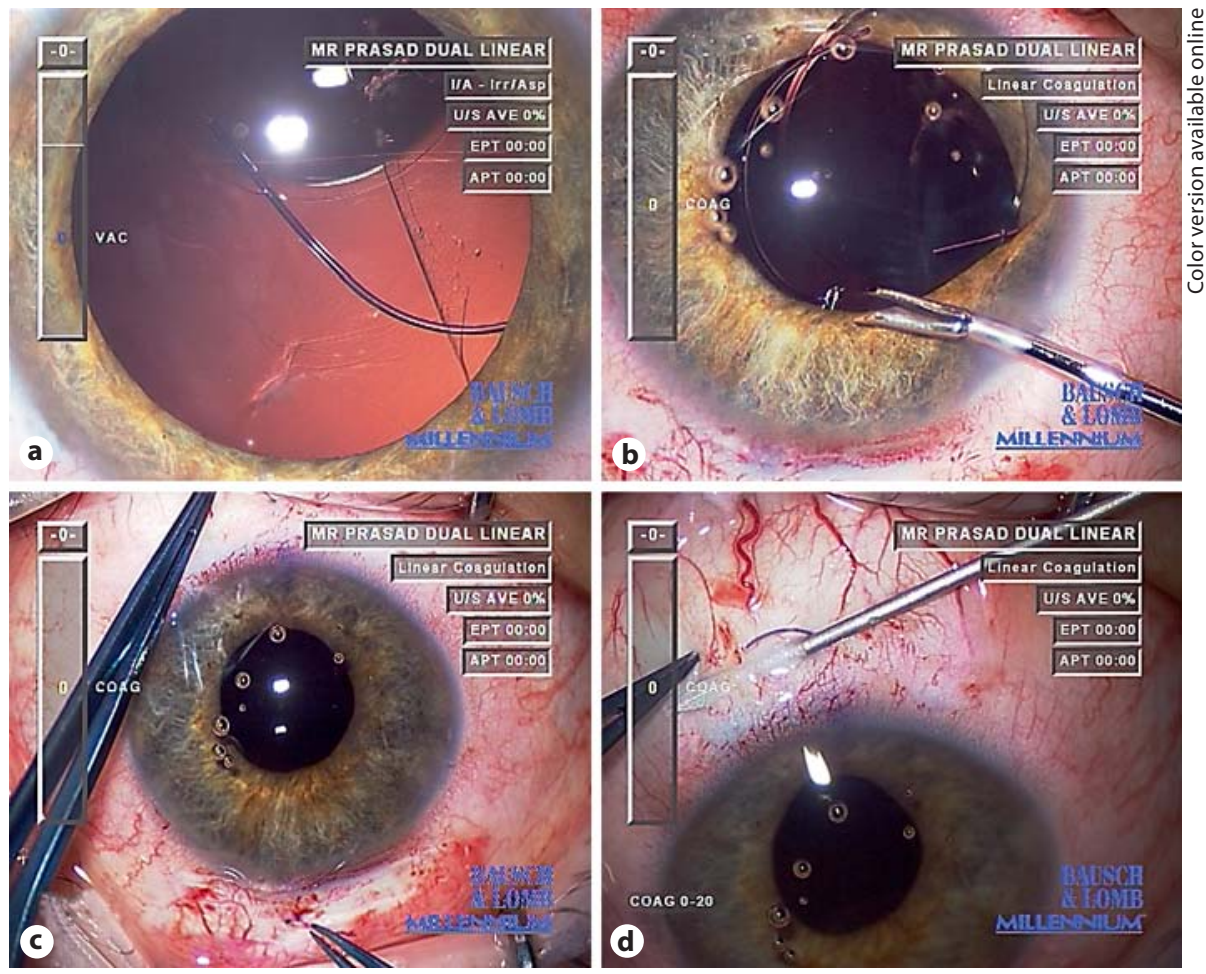

Prepare the fibrin glue as per the manufacturer's instructions and inject it under both scleral flaps. Apply local pressure over the closed flaps for approximately $20 \mathrm{~s}$, allowing the glue to stick. If the haptic extends beyond the pouch created, bury the remainder in a pocket tunnelled using a 22-gauge needle. The conjunctival peritomy can be closed with the same fibrin glue, avoiding the need for any sutures at all. No complications or redislocation of IOL was encountered in 10 eyes; however, the maximum follow-up period was only 6 weeks and longerterm follow-up data are required [12].

\section{Anterior Chamber Intraocular Lenses}

ACIOL, since their first implantation almost 60 years ago, have run out of favour due to the unacceptable rate of associated complications including pseudophakic bullous keratopathy and UGH (uveitis-glaucoma-hyphaema) syndrome $[13,14]$. Significant design changes from the closed-loop IOL are now in use and include open-loop and iris clip ACIOL. The open-loop ACIOL incorporates footplates which contact the anterior chamber angle (ideally against the scleral spur) and employ the minimal contact surface area required to ensure stability which minimises the risk of complications [15]. Improved openloop ACIOL design has incorporated anterior vaulting and flexibility, which reduces iris and corneal contact. Advantages of the ACIOL over PCIOL include the ease of insertion and relative minimal time consumption for secure IOL placement. Prospective data comparing openloop ACIOL insertion in intracapsular cataract extraction with no IOL insertion [16] indicate the risk attributable specifically to the insertion of an open-loop ACIOL. This randomised controlled trial indicated there was no statistically significant increase in patients developing cystoid macular oedema or corneal decompensation compared with no IOL, with a statistically significant increase in glaucoma escalation; however, as such a small number were affected, this was concluded unlikely to be of clinical significance. A clear description on the 10-step process for successful insertion of the angle-supported, open-loop ACIOL can be found in the article by Por and Lavin [6]. Iris claw/clip lenses provide a suitable alternative to sutureless ACIOL [17]. The IOL is fixated to the iris by hooking the midperipheral iris through the claws/ clips. This lens again has the advantages of ease of implantation and minimal time consumption. 


\section{Conclusions}

There are a limited number of clinical trials comparing ACIOL with scleral-sutured PCIOL, but the results show no significant superiority of one design over the other [18], and therefore the choice of lens and method of fixation is still open to personal preference, availability and the individual surgeon's skill set. By highlighting a few of the many methods available, we hope that we have helped in the decision-making process when confronted with these challenging cases. We believe that with currently available techniques, a minimally invasive technique of relocating an in situ IOL to a secure sulcus place- ment - or exchanging it for an appropriate PCIOL secured as per available tissue - remains the goal, and the techniques described above help us to move towards that goal.

\section{Disclosure Statement}

A.B. and C.P. have no conflicts of interest to declare. S.P. is a consultant to Bausch \& Lomb and Allergan. He has received payments for lectures by Bausch \& Lomb, and for travel, accommodation and meeting expenses by Bausch \& Lomb, Alcon, Neovista, Novartis and Allergan.

\section{References}

1 Jaycock P, Johnston RL, Taylor H, Adams M, Tole DM, Galloway P, Canning C, Sparrow JM: The Cataract National Dataset electronic multi-centre audit of 55,567 operations: updating benchmark standards of care in the United Kingdom and internationally. Eye (Lond) 2009;23:38-49.

-2 Mahmood S, von Lany H, Cole MD, Charles SJ, James CRH, Foot B, Gouws P, Shaw S: Displaced nuclear fragments into the vitreous complicating phacoemulsification surgery in the United Kingdom: incidence and risk factors. Br J Ophthalmol 2008;92:488-492.

-3 von Lany H, Mahmood S, James CR, Cole MD, Charles SJ, Foot B, Gouws P, Shaw S: Displacement of nuclear fragments into the vitreous complicating phacoemulsification surgery in the UK: clinical features, outcomes and management. Br J Ophthalmol 2008;92:493-495.

-4 Chang DF, Masket S, Miller KM, Braga-Mele R, Little BC, Mamalis N, Oetting TA, Packer $\mathrm{M}$ : Complications of sulcus placement of single-piece acrylic intraocular lenses. J Cataract Refract Surg 2009;35:1445-1458.

5 Gimbel HV, Neuhann T: Development, advantages and methods of the continuous circular capsulorhexis technique. J Cataract Refract Surg 1990;16:31-37.
6 Por YM, Lavin MJ: Techniques of intraocular lens suspension in the absence of capsular/zonular support. Surv Ophthalmol 2005; 50:429-462.

7 Hoffman RS, Fine IH, Packer M: Scleral fixation without conjunctival dissection. J Cataract Refract Surg 2006;32:1907-1912.

-8 Szurman P, Petermeier K, Aisenbrey S, Spitzer MS, Jaissle GB: Z-suture: a new knotless technique for transscleral suture fixation of intraocular implants. Br J Ophthalmol 2010;94:167-169.

-9 Scharioth GB, Gabor MD, Mitrofanis M, Pavlidis MD: Sutureless intrascleral posterior chamber intraocular lens fixation. J Cataract Refract Surg 2007;33:1851-1854.

10 Scharioth GB, Prasad S, Georgalas I, Tataru C, Pavlidis M: Intermediate results of sutureless intrascleral posterior chamber intraocular lens fixation. J Cataract Refract Surg 2010;36:254-259.

11 Fink D, Klein JJ, Kang H, Ergin MA: Application of biological glue in repair of intracardiac structural defects. Ann Thorac Surg 2004;77:506-511.

12 Agarwal A, Kumar DA, Jacob S, Baid C, Agarwal A, Srinivasan S: Fibrin glue-assisted sutureless posterior chamber intraocular lens implantation in eyes with deficient posterior capsules. J Cataract Refract Surg 2008; 34:1433-1438.
13 Sawada T, Kimura W, Kimura T, Suga H, Ohte A, Yamanishi S, Ohara T: Long-term follow-up of primary anterior chamber intraocular lens implantation. J Cataract Refract Surg 1998;24:1515-1520.

14 Auffarth GU, Wesendahl TA, Brown SJ, Apple DJ: Are there acceptable anterior chamber intraocular lenses for clinical use in the 1990 s? An analysis of 4,104 explanted anterior chamber intraocular lenses. Ophthalmology 1994;101:1913-1922.

15 Dick HB, Augustin AJ: Lens implant selection with absence of capsular support. Curr Opin Ophthalmol 2001;12:47-57.

16 Hennig A, Evans JR, Pradhan D, Johnson GJ, Pokhrel RP, Gregson RM, Hayes R, Wormald RP, Foster A: Randomised controlled trial of anterior-chamber intraocular lenses. Lancet 1997;349:1129-1133.

17 Worst JG, Los LI: Some aspects of implant surgery. Eur J Implant Refract Surg 1991;3: 157-167.

18 Wagner MD, Cox TA, Ariyasu RG, Jacobs DS, Karp CL: Intraocular lens implantation in the absence of capsular support : a report by the American Academy of Ophthalmology. Ophthalmology 2003;110:840-859. 